\title{
Artefact
}

Techniques, histoire et sciences humaines

$9 \mid 2018$

Techniques, stratégies et alimentation pour temps de guerre

\section{Aspects singuliers de la politique alimentaire de la Belgique en temps de guerre et de sortie de guerre (1914-1921 et 1939-1948)}

Some original aspects of Belgium's food policy in war and after war (1914-1921

and 1939-1948)

\section{Luis Angel Bernardo y Garcia}

\section{(2) OpenEdition}

Journals

\section{Édition électronique}

URL : http://journals.openedition.org/artefact/2604

DOI : $10.4000 /$ artefact.2604

ISSN : 2606-9245

Éditeur :

Association Artefact. Techniques histoire et sciences humaines, Presses universitaires du Midi

\section{Édition imprimée}

Date de publication : 15 mars 2019

Pagination : 15-34

ISBN : 978-2-8107-0623-5

ISSN : 2273-0753

\section{Référence électronique}

Luis Angel Bernardo y Garcia, « Aspects singuliers de la politique alimentaire de la Belgique en temps de guerre et de sortie de guerre (1914-1921 et 1939-1948) », Artefact [En ligne], 9 | 2018, mis en ligne le 04 mars 2020, consulté le 27 novembre 2020. URL : http://journals.openedition.org/artefact/2604 ; DOI : https://doi.org/10.4000/artefact.2604

\section{(c) (i) () $\Theta$}

Artefact, Techniques, histoire et sciences humaines est mise à disposition selon les termes de la Licence Creative Commons Attribution - Pas d'Utilisation Commerciale - Pas de Modification 4.0 International. 


\section{Aspects singuliers de la politique alimentaire de la Belgique en temps de guerre et de sortie de guerre (1914-1921 et 1939-1948)}

\section{Luis Angel Bernardo y Garcia}

\section{Résumé}

Entre 1914 et 1948, la Belgique connaît près de seize années de privations et de disette. Cinq crises alimentaires sont marquées par la chute brutale, voire la quasi-disparition, de ses approvisionnements extérieurs vitaux. Au cours des deux occupations, la population échappe à la famine en dépit du blocus britannique et des prédations allemandes. Lors des deux sorties de guerre, la Belgique parvient à relancer rapidement sa production industrielle et ses exportations. Ces « miracles » ne peuvent être compris qu'en analysant ces crises alimentaires dont la puissance publique, les élites dirigeantes, le monde agricole et le mouvement ouvrier ont su tirer les enseignements. La constitution de stocks de sécurité « privés » au cours de la drôle de guerre (1939-1940), le ravitaillement de la Belgique occupée (1940-1944) depuis les pays européens neutres se trouvant à l'intérieur du blocus allié ou encore la « politique d'abondance » de l'offre de produits alimentaires après-guerre (1945-1948) en constituent de parfaites illustrations.

\section{Mots-clés}

Belgique, politique alimentaire, premier après-guerre (1918-1921), Première Guerre mondiale, second après-guerre (1945-1948), Seconde Guerre mondiale

99 Luis Angel Bernardo y Garcia, « Aspects singuliers de la politique alimentaire de la Belgique en temps de guerre et de sortie de guerre (1914-1921 et 1939-1948) », Artefact, 9, 2018, p. 15-34. 


\section{Some original aspects of Belgium's food policy in war and after war (1914-1921 and 1939-1948)}

\section{Abstract}

From 1914 to 1948, Belgium experienced nearly sixteen years of deprivation and scarcity. Five food crises were caused by the sudden falling and even disappearing of its vital external supplies. During both occupations, the population hardly avoided famine despite the British blockade and the German predations. During the two post-wars, Belgium managed to restore quickly its industrial production and its exports. These "miracles" can only be understood by a close analysis of these food crises from which the State, the establishment, the agricultural world and the labour movement learned lessons. The constitution of "private" security stocks during the phoney war (1939-1940), the relief supply of occupied Belgium from the neutral European countries within the Allied blockade (1940-1944) or the post-war "Policy of abundance" for food products supply provide (1945-1948) 16 perfect illustrations.

\section{Keywords}

Belgium, First Postwar (1918-1921), First World War, Food Policy, Second Postwar (1945-1948), Second World War 


\section{Deux occupations (1914-1918 et 1940- 1944) et deux sorties de guerre (1918- 1921 et 1944-1948)}

Au cours de la première moitié du $\mathrm{xx}^{\mathrm{e}}$ siècle, la Belgique est le pays le plus densément peuplé au monde ${ }^{1}$. Près de $60 \%$ de la population est urbaine $^{2}$ et la moitié est concentrée dans des agglomérations d'au moins 25000 habitants " où les ressources alimentaires familiales sont nulles ou à peu près nulles ${ }^{3} »$. Depuis le $\mathrm{XIX}^{\mathrm{e}}$ siècle, la Belgique exporte essentiellement de la "valeur ajoutée ", c'est-à-dire du travail ${ }^{4}$. En quelque sorte, la fourmilière belge vend au monde ses produits industriels semi-finis pour importer ses matières premières ainsi que le pain blanc (à base de froment et bluté à $72 \%$ ) que les ouvriers ont obtenu de pouvoir consommer depuis la fin du xix siècle. En 1939, la Belgique achète à l'étranger $70 \%$ de la valeur énergétique de la nourriture consommée si l'on inclut les aliments du bétail ${ }^{5}$. Un quart des importations de froment en Europe continentale est l'œuvre de la seule et modeste Belgique ${ }^{6}$. Cette dépendance à l'égard de l'étranger n'existe à un même degré que dans un autre pays de vieille industrie capitaliste, à savoir la Grande-Bretagne ${ }^{7}$.

Dans le cas des deux occupations comme pour les deux sorties de guerre, on pourrait parler d'une Belgique " miraculée " au vu des difficultés structurelles qui affectent son économie libre-échangiste et qui ont pu - ou su être surmontées. À vrai dire, la Providence n'y est pas pour grand-chose, à

1. Archives générales du Royaume (désormais AGR), Cabinets des ministres du Ravitaillement (désormais CABRAV), 117, Note sommaire - Document portant le $\mathrm{n}^{\circ}$ 4. Principales phases de l'évolution de la politique générale du Département depuis la Libération, Bruxelles, 27 novembre 1947, p. 5.

2. AGR, CABRAV, 1663, Note concernant le ravitaillement de la Belgique, s.d. [février 1947], p. 1.

3. AGR, CABRAV, 137, Projet de discours de Mathieu, Bruxelles, 18 octobre 1946, p. 2.

4. Isabelle Cassiers, Philippe Ledent, La Banque nationale de Belgique. 1939-1971, vol. 4, Politique monétaire et croissance économique en Belgique à l'ère de Bretton Woods (1944-1971), Bruxelles, Banque nationale de Belgique, 2005, p. 48-49.

5. AGR, CABRAV, 117, Note sommaire, p. 1. AGR, Archives du sous-secrétariat d'État au Ravitaillement, 24, dossier "Divers alim. ", Note sur le problème des secours alimentaires à la Belgique envisagé du point de vue de la politique de guerre, 28 juin 1941, p. 2.

6. AGR, CABRAV, 735, Emergency Conference on European Cereals Supplies held in London from 3rd to 6th April 1946, Londres, 1946, p. 47-51.

7. Lizzie Collingham, The Taste of War: World War II and the Battle for Food, New York, Penguin Books, 2012, p. 19. Eric J. Новsваwм, Lère des empires 1875-1914, Paris, Pluriel, 2007, p. 53-54. 
l'exception des pêches exceptionnelles de harengs, sous la seconde occupation et à la Libération, qui constituèrent un apport inespéré de protéines animales pour la population sous-alimentée. La survie alimentaire doit davantage à la pugnacité des élites administratives, financières et industrielles dans Bruxelles occupée ainsi qu'à celle du gouvernement en exil au Havre puis à Londres, à l'" économie morale " populaire et patriotique opposée au marché noir mais aussi, et surtout, au bon vouloir de la puissance occupante et des puissances alliées tutélaires.

Au cours des deux occupations, les pouvoirs exécutif et législatif doivent quitter le territoire national pour échapper à l'ennemi. Avec l'accord de la puissance occupante, l'espace public - en termes de "gouvernance » et d'autorité morale légitime à défaut d'être légale - est occupé par des élites financières et industrielles. Celles-ci prennent des mesures d'ordre monétaire et organisent les principes des approvisionnements extérieurs ${ }^{8}$. À l'estompement des normes, à la " brutalisation " des rapports sociaux et économiques, à l'appât du gain et à la disqualification de la puissance publique, la demande ouvrière répond par une exigence de justice et d'équité. Cette "économie morale populaire " s'exprime par des manifestations de femmes et des grèves sauvages, voire des actions violentes contre les biens et les accapareurs et spéculateurs mais aussi contre l'arbitraire des notables locaux. En outre, au cours de la seconde occupation, dans le cadre des transactions clandestines, la défense du " prix juste " est organisée par la Résistance armée qui use de menaces et de violences à l'encontre de fermiers et commerçants accapareurs'. À l'image de l'économie morale populaire, une économie clandestine qualifiable de " morale et patriotique " enfreint la législation d'avant-guerre, celle mise en œuvre en Belgique occupée par le Comité des secrétaires généraux et les ordonnances allemandes. Sans doute, cette action alimentaire illégale promue par les

8. Sophie de Schaepdrijver, La Belgique et la Première Guerre mondiale, Bruxelles, Archives et Musée de la Littérature, 2004 ; Ginette Kurgan-Van Hentenryk, Max-Léo Gérard. Un ingénieur dans la cité (1879-1955), Bruxelles, Éditions de l'Université de Bruxelles, 2010.

9. En 1996, John Horne propose pour la Grande Guerre une approche de la politique alimentaire qu'il qualifie de "novatrice " sur la base de l'« économie morale " populaire conceptualisée par Edward P. Thomson dans le cas de l'Ancien Régime. John HoRne, "Introduction. État, sociétés et "économie morale" : l'approvisionnement des civils pendant la guerre de 1914-1918 ", Guerres mondiales et conflits contemporains, $\mathrm{n}^{\circ} 183$, juillet 1996, p. 6. Cette approche a été appliquée dans notre étude diachronique des deux occupations et sorties de guerre en Belgique. L. A. Bernardo y Garcia, Le Ventre des Belges, op. cit. 
notables repose-t-elle sur le même «état de nécessité » invoqué par les élites financières et industrielles pour justifier leur politique pragmatique de travail et de production au profit de l'effort de guerre du III ${ }^{e}$ Reich ${ }^{10}$.

En 1914-1918, la survie alimentaire du pays est assurée par les importations transatlantiques de la Commission for Relief in Belgium dirigée depuis Londres par l'Américain Herbert Hoover. Ces importations sont financées par des dons privés mais aussi et surtout par le gouvernement belge exilé au Havre grâce aux prêts accordés par la France, la Grande-Bretagne puis les États-Unis ${ }^{11}$. De fait, la puissance occupante externalise habilement le ravitaillement général du pays qui incombe à l'Entente, aiguillonnée par l'opinion publique américaine et Hoover. Grâce à l'offre légale et clandestine belge, l'Allemagne poursuit et amplifie ses prélèvements sur la production agricole indigène y compris les denrées déficitaires. Comme le reconnaît dans ses mémoires l'ancien général en chef des armées allemandes, Erich Ludendorff, la Belgique contribua " puissamment » au ravitaillement du Reich $^{12}$. La Grande-Bretagne, flouée, saura s'en souvenir.

Au cours de la seconde occupation (mai 1940-septembre 1944), la politique de travail et de production au profit du Reich prônée par les élites face au blocus intraitable britannique permet à la Belgique d'organiser des importations de céréales depuis l'Europe sous le joug allemand qui assurent la soudure ${ }^{13}$. Ces importations alimentaires ponctuelles, payées rubis sur l'ongle, ne constituent pas les contreparties attendues par les notables pour les prestations belges dans le cadre de leur politique dite

\footnotetext{
10. L. A. Bernardo y Garcia, Le Ventre des Belges, op. cit., p. 234.

11. Au total, la Commission for Relief in Belgium parvient à importer quelque 4,8 millions de tonnes de marchandises pour une valeur de 4 milliards de francs. Leen VAN Molle, Chacun pour tous. Le Boerenbond belge 1890-1990, Louvain, Leuven University Press, 1990, p. 141.

12. Erich Ludendorff, Souvenirs de guerre, Paris, Payot, t. 1, 1920, p. 381-382. L'essentiel des transactions organisées par l'occupant étant clandestines, il demeure difficile d'estimer l'ampleur des prélèvements sur les maigres ressources indigènes. Néanmoins, la duplicité de la puissance occupante « cabre " à plusieurs reprises la Grande-Bretagne qui tente de mettre fin au ravitaillement transatlantique. Celui-ci est sauvé à chaque fois par l'opinion publique américaine et par le gouvernement belge en exil qui n'hésite pas à mettre son engagement militaire dans la balance. L. A Bernardo y Garcia, Le Ventre des Belges, op. cit., p. 82.

13. Entre 1940 et 1944, le Comité des Secrétaires généraux, réunissant les plus hauts fonctionnaires de chaque département et remplaçant les ministres absents, importe depuis des pays amis ou occupés par le Grand Reich allemand 850370 t de céréales panifiables. Jean Colard, L'Alimentation de la Belgique sous l'Occupation Allemande 1940-1944, Louvain, Nouvelles Publications Universitaires, 1945 , p. 55 et 56.
} 
"du moindre mal ${ }^{14}$ ». Elles écartent le spectre de la famine qui s'est abattue sur la malheureuse Grèce et qui constitue une arme supplémentaire pour le Reich dans sa guerre d'anéantissement à l'Est ${ }^{15}$. La disette, les carences alimentaires consécutives et les maladies liées à celles-ci (œdème, rachitisme, tuberculose, etc.) pèsent lourdement sur la santé des Belges occupés, surtout pour les catégories les plus fragiles de la population dont le monde ouvrier et la petite bourgeoisie au faible pouvoir d'achat. Le coût humain de cette disette s'avère toutefois difficile à estimer. À l'image de la première occupation, la seconde connaît une baisse singulière de la mortalité infantile par rapport au temps de paix grâce à l'action hygiéniste, médicale et nutritionnelle des organisations caritatives et philanthropiques nationales. Ce résultat doit être toutefois nuancé en raison de la baisse inquiétante du nombre de naissances ainsi que du poids et de la taille des nouveau-nés ${ }^{16}$. Les richesses minérales et végétales stratégiques du Congo ainsi que les réserves en or et en devises assurent au gouvernement belge exilé à Londres le nihil obstat de la Grande-Bretagne pour un ravitaillement complémentaire réduit à partir du Portugal neutre mais anglophile ${ }^{17}$.

La Belgique ne semblait donc guère armée pour supporter deux occupations et deux longues sorties de guerre. Pourtant, à deux reprises, elle survit au joug allemand et parvient à relancer rapidement après-guerre sa production industrielle et ses exportations. Il faut rappeler que la Belgique fut le seul État, avec le Grand-Duché de Luxembourg, à connaître en Europe occidentale deux occupations ${ }^{18}$ et dès lors deux libérations ${ }^{19}$. Entre 1914

14. Étienne Verhoeyen, "Moindre mal et bonne réputation ", Jours de Guerre, Bruxelles, Crédit communal, 1993, p. 17-33.

15. Pavel Polian, " La violence contre les prisonniers de guerre soviétiques dans le III" Reich et en URSS ", dans Stéphane Audoin-Rouzeau, Annette Becker, Christian Ingrao, Henry Rousso (dir.), La violence de guerre 1914-1945: approche comparée des deux conflits mondiaux, Bruxelles, Complexe, 2002, p. 117-134.

16. Peter Scholliers, "Santé publique ", in Dictionnaire de la Seconde Guerre mondiale en Belgique, Bruxelles, André Versailles, 2008, p. 403-405. Claudine Marissal, "Enfance (Protection de l')", dans Dictionnaire de la Seconde Guerre mondiale en Belgique, op. cit., p. 161 ; id., "Protéger l'enfance : l'Euvre Nationale de l'Enfance en Belgique (1919-1940)", Histoire \& Sociétés. Revue européenne d'histoire sociale, $\mathrm{n}^{\circ} 15,2005$, p. 8-15.

17. Joaquim da Costa Leite, "Neutrality by Agreement: Portugal and the British Alliance in World War II ", American University International Law Review, 14, n 1, 1998, p. 188 et 190-192. 18. Sur la plus grande partie du territoire.

19. Benoît Majerus, Charles Roemer, "Luxembourg ", 1914-1918-online. International Encyclopedia of the First World War, Berlin, 2015. https://encyclopedia.1914-1918-online.net/ article/luxembourg (consulté le 19 décembre 2016). 
et 1948, le pays expérimente donc neuf années de sous-alimentation sévère, sous une occupation étrangère et à sa libération, et près de sept " années maigres $^{20}$ » d'après-guerre.

Comme le rappelait déjà le ministre communiste du Ravitaillement Edgar Lalmand au mois d'octobre 1946 devant des parlementaires britanniques dubitatifs : "les difficultés de ravitaillement de mon pays [sont] spécifiques et notre expérience le fruit de plus de 10 années de disette vécue en une période de 30 ans ${ }^{21}$ ». Entre 1939 et 1948, la puissance publique, les élites dirigeantes, le monde agricole et le mouvement ouvrier savent tirer les enseignements des crises alimentaires précédentes marquées par une chute brutale - voire une quasi-disparition - des importations de céréales - vitales pour les populations -, par des prélèvements allemands et par l'affaiblissement consécutif des forces de travail indispensables à la restauration économique du pays.

La politique alimentaire en temps de pénurie constitue l'ensemble des compétences exercées par la puissance publique. Elle repose sur deux piliers indissociables à savoir, la politique des prix et salaires et le ravitaillement général du pays proprement dit. Le premier pilier a pour objectif de contenir l'inflation des prix de détail et une pression conséquente à la hausse des salaires. Le second doit assurer une répartition équitable des ressources disponibles. La singulière politique alimentaire de la Belgique mise en œuvre entre 1914 et 1921 puis entre 1939 et 1948 a été abordée inégalement par les historiographies belge et internationale. En outre, aucune approche diachronique des deux temps d'occupation (1914-1918 et 1940-1944) et des sorties de guerre (1918-1921 et 1944-1948) n'a été proposée à l'exception de notre thèse de doctorat soutenue en $2015^{22}$. Tantôt la recherche s'est focalisée sur le ravitaillement de la première ou de la seconde occupation ; tantôt elle s'est penchée, pour les deux libérations et après-guerres respectives, sur la politique des prix et salaires - et de façon plus générale sur les questions financières et monétaires -, détachée de son binôme à savoir le ravitaillement ${ }^{23}$.

20. S. de Schaepdrijver, La Belgique et la Première Guerre mondiale, op. cit.

21. AGR, CABRAV, 1663, discours du ministre Lalmand à la Fondation universitaire lors de la visite de parlementaires britanniques, 18 octobre 1946, p. 1.

22. L. A. Bernardo y Garcia, Le Ventre des Belges, op. cit.

23. Pour une approche détaillée de l'historiographie belge et internationale sur les politiques alimentaires belges des temps de (sortie de) guerre, le lecteur est invité à consulter librement en ligne le 


\section{Les « stocks de sécurité " privés (1939- 1940)}

Au cours de la seconde occupation (1940-1944) et du second immédiat après-guerre (1945-1948), la Belgique, à l'image de ses voisins, est soumise à une "économie de disette ${ }^{24}$ ». Afin d'assurer le ravitaillement alimentaire de la population et le maintien d'une activité économique, la puissance publique est contrainte d'organiser l'économie nationale traditionnellement acquise à la liberté du marché. La Belgique agrippée à sa neutralité déploie déjà, dès la drôle de guerre, différentes stratégies pour se dresser face non seulement au blocus anglo-français mais aussi à la menace d'une seconde invasion et donc de nouvelles prédations allemandes massives sur ses maigres ressources alimentaires ${ }^{25}$.

La menace de guerre entraîne rapidement la mobilisation des acteurs du secteur alimentaire. Celle-ci repose essentiellement sur la constitution de stocks dits " de sécurité ${ }^{26}$ ". Ceux-ci sont destinés à compenser la baisse ponctuelle et imprévue des importations en cas de blocus mais aussi à apporter les premiers approvisionnements à la population dans le cas d'une nouvelle invasion du territoire national. Dès 1932, le Conseil des ministres s'accorde avec la Commission permanente de mobilisation de la nation sur la nécessité de garantir les approvisionnements du pays en blé « en temps de guerre ${ }^{27}$ ". Pourtant, le stockage des matières premières alimentaires stratégiques reste très limité en $1938^{28}$. Les réserves de blés exotiques dans la métropole anversoise correspondent tout au plus à une consommation d'une semaine et demie tout comme les stocks réalisés par les meuneries ${ }^{29}$.

chapitre correspondant de la thèse de doctorat sur le site internet de l'Université libre de Bruxelles : http://difusion.ulb.ac.be/vufind/Record/ULB-DIPOT:oai:dipot.ulb.ac.be:2013/209052/Holdings 24. "Les industries belges de 1939 à 1945 ", Bulletin d'Information et de Documentation, $\mathrm{XXI}^{\mathrm{e}}$ année, vol. 2, n 3, 1946, p. 78.

25. L. A.Bernardo y Garcia, Le Ventre des Belges, op. cit., p. 133-137.

26. AGR, CABRAV, 1663, Note concernant le ravitaillement de la Belgique, p. 2.

27. AGR, procès-verbaux des séances du conseil des ministres (désormais PVCM), séance du 18 août 1932.

28. AGR, PVCM, séance du 9 mars 1939.

29. AGR, PVCM, séance du 27 septembre 1938. 
Dès juin 1939, la puissance publique opère par voie de contrainte sur les meuniers $^{30}$, fabricants de margarine ${ }^{31}$, importateurs de viande congelée ${ }^{32}$. Avec l'état de guerre en Europe, les mesures s'étendent à tous les importateurs, transformateurs et distributeurs de denrées alimentaires. La constitution des "stocks de sécurité " repose sur une approche qui se distingue singulièrement des pratiques en cours en Grande-Bretagne ou encore aux Pays-Bas puisque la Belgique privatise en quelque sorte ses réserves alimentaires stratégiques ${ }^{33}$. Concrètement, tout importateur, commerçant et transformateur est tenu de détenir à ses frais une réserve de marchandises faisant l'objet de son négoce, proportionnelle aux besoins de sa clientèle pendant une période de référence ${ }^{34}$.

Aux yeux de la puissance publique, le stockage privé présente différents avantages. Tout d'abord, il évite l'intervention financière de l'État confronté à la mobilisation coûteuse de l'armée ${ }^{35}$. La politique alimentaire assume les convictions libres-échangistes de tous les acteurs économiques tout en garantissant - théoriquement du moins - les intérêts des consommateurs et futurs rationnaires en cas d'invasion du territoire ${ }^{36}$. L'État peut constituer à moindres frais sur tout le territoire des stocks alimentaires de toutes tailles rapidement accessibles aux pouvoirs publics ${ }^{37}$ et aux consommateurs ${ }^{38}$. Il se prémunit des risques de destruction complète après les opérations de guerre. La solution gouvernementale devrait poser un obstacle juridique à la confiscation éventuelle par l'occupant puisque s'agissant de stocks privés il ne peut s'agir de "butin de guerre ${ }^{39}$ ». Enfin, par leur "dissémination extrême ${ }^{40}$ ", les stocks entravent leur réquisition éventuelle par l'occupant ${ }^{41}$.

30. Arrêté royal du 22 juin 1939, Moniteur belge, 28 juin 1939.

31. Arrêté royal du 17 juillet 1939, Moniteur belge, 22-23 juillet 1939.

32. Arrêté royal du 9 août 1939, Moniteur belge, 21-22 août 1939.

33. AGR, CABRAV, 224, Historique du département du Ravitaillement, Bruxelles, 24 juillet 1946, p. 1 bis et 2 .

34. AGR, CABRAV, 1629, Résumé d'un entretien avec Monsieur Demaree Bes, p. 2.

35. AGR, CABRAV, 1663, Note concernant le ravitaillement de la Belgique, p. 2.

36. GR, CABRAV, 1654, Du ravitaillement de la Belgique de 1939 à 1947, p. 2.

37. Fernand Baudhuin, L'économie belge sous l'occupation 1940-1944, Bruxelles, Bruylant, 1945, p. 33.

38. AGR, CABRAV, 137, Projet de discours de Mathieu, Bruxelles, 16 octobre 1946, p. 2.

39. F. BAudhuin, L'économie belge sous l'occupation 1940-1944, op. cit., p. 56.

40. AGR, CABRAV, 1654, Du ravitaillement de la Belgique de 1939 à 1947, p. 3.

41. Ibid., p. 2. 
Au 10 mai 1940, la Belgique dispose de réserves alimentaires « dont l'importance n'avait eu d'autres limites que la durée de conservation des produits ou les possibilités financières de l'industrie et du commerce ${ }^{42}$ ». Ces réserves s'élèvent à $330000 \mathrm{t}$ dans le pays et entre 250000 et $300000 \mathrm{t}$ en route pour Anvers soit le triple des réserves habituelles du temps de paix ${ }^{43}$. Elles assurent la soudure sur la base d'une consommation unitaire de 350 à $400 \mathrm{~g}$ de pain bluté à $82 \%{ }^{44}$. La place d'Anvers, qui a pour obligation de stocker 60000 t, en a stocké plus de 100000 majoritairement logées en allèges ${ }^{45}$. La meunerie industrielle a accumulé $80000 \mathrm{t}$ dans les moulins et 20000 sur des allèges et dispose d'un fonds de roulement de $30000 \mathrm{t}$. Les approvisionnements des boulangers sont portés de quinze jours à un mois ${ }^{46}$. À ces réserves stratégiques s'ajoutent les "stocks énormes " mais difficilement chiffrables constitués par les ménages ${ }^{47}$.

Lors de l'invasion allemande, des stocks de sécurité seront pillés ou détruits $^{48}$. À vrai dire, la plupart échappent aux filets allemands contrairement aux grands stocks constitués par la puissance publique en France ou aux Pays-Bas ${ }^{49}$. La destruction prudente de l'inventaire par le département du Ravitaillement y contribue puissamment. Ces stocks de sécurité disparaissent aussi de la surveillance du Comité des secrétaires généraux qui administre le pays occupé et doit assurer son ravitaillement ${ }^{50}$. Une faible partie est consommée les premiers jours de l'occupation dans le cadre du commerce légal, le reste au volume incertain constitue une partie des fameux "stocks non déclarés " qui suscitent la convoitise allemande tout au long de l'occupation ${ }^{51}$.

42. AGR, CABRAV, 1663, Discours du ministre du Ravitaillement à la Fondation universitaire devant des parlementaires britanniques, p. 1.

43. F. Baudhuin, L'économie belge sous l'occupation 1940-1944, op. cit., p. 57. L. VAN Molle, Chacun pour tous, op. cit., p. 325.

44. AGR, CABRAV, 1629, Résumé d'un entretien avec Monsieur Demaree Bes, p. 3.

45. Ibid.

46. F. BAudhuin, L'économie belge sous l'occupation 1940-1944, op. cit., p. 57.

47. Ibid., p. 58.

48. Alain Colignon, "Agriculture", in Dictionnaire de la Seconde Guerre mondiale en Belgique, op. cit., p. 25.

49. Surtout ceux de petites tailles et en possession des distributeurs et commerçants.

50. Le Comité des secrétaires généraux ne parvient à récupérer que $65000 \mathrm{t}$. Alain Colignon, "Secours d'hiver, Secours d'Hitler ", Jours de guerre, Bruxelles, Dexia, 1992, p. 65 ; Jules GérardLibois, José Gotovitch, L'an 40, la Belgique occupée, Bruxelles, CRISP, 1971, p. 330.

51. Paul SAnders, Histoire du marché noir 1940-1946, s. 1., 2001, p. 234-235 et 239 ; Étienne Verhoeyen, La Belgique occupée. De l'An 40 à la Libération, Bruxelles, De Boeck-Wesmael, 1994, p. 222. 


\section{Conserves portugaises, huile de foie de poisson et « banacao numéro 2 " (1940-1944)}

Au cours de la Seconde Guerre mondiale, le spectre de la « famine ${ }^{52}$ » hante le gouvernement belge en exil à Londres. En raison de sa dépendance existentielle aux approvisionnements extérieurs, la Belgique est le pays le plus mal loti d'Europe occidentale occupée et l'attention portée par les opinions publiques britannique ou américaine à son sort - aux côtés de la malheureuse Grèce - n'est pas seulement due à la propagande belge ou au poids dans les médias américains d'un Herbert Hoover qui reprend du service ${ }^{53}$. Tout comme le Danemark ${ }^{54}$, les Pays-Bas parviennent à assurer un rationnement alimentaire et un contrôle efficace des prix en dépit du marché noir et des prélèvements allemands. Le Hongerwinter met brutalement fin à une situation relativement privilégiée. Au cours des premiers mois de l'année 1945, la famine y emporte une dizaine de milliers de civils ${ }^{55}$. Aux côtés des notables du pays occupé, le gouvernement belge présidé par Hubert Pierlot appréhende pour l'après-guerre de graves conséquences en matière de santé publique hypothéquant toute restauration économique mais aussi sociale et morale du pays. Aussi le gouvernement belge à Londres tente-t-il rapidement d'organiser un "ravitaillement de guerre ${ }^{56}$ " à destination du pays occupé ${ }^{57}$. L'objectif initial est de renouveler l'expérience de

52. AGR, papiers Hubert Pierlot, 351, Radio-Canada, le 3 mai 1941, s.d., p. 4.

53. James H. George, "Another Chance: Herbert Hoover and Ward War II Relief », Diplomatic History, $\mathrm{n}^{\circ} 16,1992$, p. 389-407.

54. Mogen R. Nissen, "Danish Food Production in the German War Economy ", in Frank Trentmann et Fleming Just (dir.), Food and Conflicts in the Age of two World Wars, Palgrave Macmillan, 2006, p. 172-192.

55. La famine est causée par un hiver rigoureux et les inondations défensives provoquées par les armées allemandes en retraite disloquant les voies de communication et isolant les villes affamées des campagnes. Hein A. M. Klemann, Nederland. 1938-1948. Economie en samenleving in jaren van oorlog en bezetting, Amsterdam, Boom, 2002, p. 463-468.

56. L'expression survient pour la première fois dans les sources consultées en septembre 1940 dans une lettre du ministre des Finances, Camille Gutt, adressée à l'homme d'affaires et futur sous-secrétaire d'État au Ravitaillement au sein du gouvernement belge en exil à Londres Raoul Richard. Elle désigne concrètement l'organisation d'un ravitaillement alimentaire depuis le monde libre à destination du pays occupé. Centre d'études et de documentation Guerre et Sociétés contemporaines (désormais CEGES), papiers Raoul Richard (désormais PR), 88, Gutt à Richard, Londres, 3 septembre 1940 .

57. L. A. Bernardo y Garcia, Le Ventre des Belges, op. cit., p. 151-178. 
la Première Guerre mondiale avec un ravitaillement collectif et massif à partir des États-Unis neutres - mais amicaux - et sous leur contrôle.

Le gouvernement belge rencontre rapidement l'opposition britannique à toute levée du blocus maritime et l'entreprise capote avec l'entrée en guerre des Américains au mois de décembre 1941. Les deux pays se refusent à toute levée partielle du blocus maritime en faveur des catégories les plus fragiles. Le Britannique Hugh Dalton, Minister of Economic Warfare, invoque dès octobre 1940 les enseignements néfastes de la première occupation : "l'étude, à laquelle il avait procédé, de l'intervention de la Commission for Relief in Belgium dans la dernière guerre, l'avait convaincu que, malgré ses motifs humanitaires élevés, cette intervention n'avait fait que retarder la débâcle allemande et, en conséquence, prolonger cruellement les souffrances en pays occupés ${ }^{58}$ ". Pour des considérations géostratégiques, les grands alliés anglo-saxons se montrent pourtant plus prévenants à l'égard de la France collaborationniste du maréchal Philippe Pétain, grande productrice de blé avant-guerre ${ }^{59}$, qu'à l'égard de « loyaux ${ }^{60}$ " petits alliés structurellement déficitaires comme la Belgique soumise à une disette ou la Grèce réduite à l'état de famine ${ }^{61}$. Il est vrai que l'image de la Belgique est sérieusement écornée par la capitulation royale du 28 mai 1940, les errements gouvernementaux après la défaite de la France et l'attentisme du Palais $^{62}$ tout au long de l'occupation. Les initiatives intempestives mais sincères de Herbert Hoover, isolationniste et adversaire acharné de Roosevelt, en faveur d'un ravitaillement transatlantique, alourdissent le passif belge ${ }^{63}$.

Face au refus réitéré des Britanniques et des Américains, le gouvernement belge à Londres se recentre rapidement sur un " ravitaillement de guerre " à la fois individuel et collectif de moindre ampleur à partir de son importante représentation diplomatique au Portugal. Le gouvernement belge à Londres est le seul petit allié à obtenir une brèche durable dans le blocus britannique. À la différence des autres petits alliés polonais, yougoslave ou

58. CEGES, PR, 93, Dalton à Gutt, Londres, 11 octobre 1940.

59. En 1938, la France intervient à raison de $15 \%$ juste après les $20 \%$ de l'Allemagne dans la production agricole européenne estimée à 12,5 milliards de dollars. Michel-Pierre CHÉLINI, "La crise du ravitaillement en Europe 1944-1949", dans Dominique Barjot, Rémi Baudouï et Daniel Voldman (dir.), Les reconstructions en Europe (1945-1949), Bruxelles, Complexe, 1997, p. 158.

60. AGR, PP, 51, Spaak à Van der Straeten Ponthoz, 18 septembre 1942.

61. Mark Mazower, Dans la Grèce d'Hitler (1941-1944), Paris, Belles Lettres, 2002.

62. À savoir le Roi " prisonnier " au Palais de Laeken et son entourage.

63. L. A.Bernardo y Garcia, Le Ventre des Belges, op. cit., p. 160. 
encore néerlandais, il a les moyens financiers de sa politique et dispose des atours congolais. Les expéditions vers le pays occupé s'effectuent sous le couvert de la Commission mixte de secours de la Croix-Rouge internationale (CroixMixt) sise à Genève ${ }^{64}$. En janvier 1941, le consul d'Allemagne à Genève remet à CroixMixt un document garantissant la non-réquisition par les troupes et fonctionnaires allemands des vivres importés officiellement par ses soins en Belgique occupée ${ }^{65}$. Le même mois, le ministère des Affaires étrangères du Reich donne l'assurance que les secours ne seront pas déduits des rations alimentaires auxquelles la population a droit ${ }^{66}$.

"Le Portugal est sans contredit appelé à jouer le premier rôle, si pas le seul, dans les secours à apporter à la Belgique ${ }^{67}$. " Ce constat exprimé publiquement dès le 18 août 1940 à Lisbonne est curieusement celui d'un simple ressortissant belge, le docteur René Reding, un cancérologue réputé, agrégé de l'université de Bruxelles ${ }^{68}$. Le projet de Reding s'inscrit dans le vaste élan de solidarité patriotique qui saisit la plupart des expatriés dans le monde libre. La petite colonie belge au Portugal se distingue de ses consœurs par l'ébauche d'un ravitaillement limité mais collectif et régulier à destination du pays occupé. Le premier projet d'aide médicale ne parvient pourtant pas à lever les restrictions imposées par le blocus sur les aliments ou les médicaments dits " de protection » (huile de foie de morue, levure de bière, etc.) qui seraient importés des États-Unis ${ }^{69}$.

La proposition de Reding d'étendre les envois aux produits alimentaires portugais est finalement acceptée à condition que les « produits, dans leur

64. À l'automne 1940, le Comité international de la Croix-Rouge et la Ligue des Sociétés de la Croix-Rouge décident de conjuguer leurs efforts afin de créer cet organisme conjoint chargé de venir en aide aux populations civiles victimes de guerre, particulièrement aux femmes et aux enfants. AGR, Cabinets du Premier ministre Hubert Pierlot à Londres (désormais PML), 500, L'œuvre de la Commission mixte de Secours de la Croix-Rouge internationale en faveur des femmes et des enfants victimes de guerre, Genève, avril 1942, p. 3.

65. CEGES, papiers André de Kerchove (désormais PDK), 56 bis, Consulat d'Allemagne au Comité international de la Croix-Rouge et à la Ligue des sociétés de Croix-Rouge, Genève, 11 janvier 1941. 66. AGR, PML, 513, Confidentiel. Circulaire d'information n ${ }^{\circ} 1$. Organisation des Secours destinés à la population et aux prisonniers de guerre, Londres, 16 octobre 1941, p. 5.

67. René Reding, Le ravitaillement de la Belgique à partir du Portugal au cours de la guerre 19401945. Rapport sur l'activité de l'Aide médicale à la Belgique, s. l., Imprimerie des Éditeurs, 1962, p. 7. 68. Le médecin biologiste belge est directeur de l'Institut de recherches cancérologiques, Fondation Yvonne Boël et agrégé de l'Université de Bruxelles. Reding travaille alors au Centre anticancéreux de Lisbonne et à l'Institut portugais de Haute Culture. Ibid., p. 10.

69. Ibid., p. 11. 
nature et leur emballage, soient originaires de pays situés en dedans du blocus $^{70} »$. L'Aide médicale à la Belgique ne peut envoyer de vivres d'origine portugaise «si de son côté, le Portugal importait des produits de même nature ${ }^{71}$ ». L'ironie veut que les conserves de poisson à l'huile ne soient pas soumises à licence d'exportation mais les Britanniques interdisent leur envoi parce que l'emballage en fer-blanc provient dans l'immense majorité des cas des pays en dehors du blocus. Aussi, l'Aide médicale s'efforcet-elle de trouver des solutions alternatives afin d'assurer l'expédition de conserves de poisson tant recherchées par les organismes caritatifs et philanthropiques du pays occupé.

L'Aide médicale est aussi confrontée au tonnage limité des quantités de produits disponibles sur le marché portugais et non soumis aux restrictions des règles du blocus. Aussi, s'applique-t-elle à expérimenter l'élaboration de produits jusqu'alors non commercialisés pour augmenter le tonnage des produits pouvant être expédiés en Belgique ${ }^{72}$. Ceux-ci sont composés de poissons en saumure ou frits à l'escabèche, conservés dans des barils en bois, de la sauce tomate " à pourcentage élevé d'huile d'olive ", d'une farine lactée baptisée «banacao numéro 2 " et de pâte de poissons à l'huile contenue en flacons de verre ${ }^{73}$. René Reding brevette en 1941 un système novateur de stérilisation de poisson à l'huile en bocaux de verre. Ce brevet est vendu à l'industrie locale de la conserverie pour la modique somme de 500000 escudos soit 50000 livres sterling de l'époque ${ }^{74}$.

Dès la fin de l'année 1940, le gouvernement belge décide de mettre la main au portefeuille et s'attache à imposer, non sans mal, son leadership à la structure d'aide humanitaire embryonnaire privée mise en place à Lisbonne. Il s'agit de coordonner et d'unifier le ravitaillement de la Belgique par l'Europe " c'est-à-dire en deçà de la ligne du blocus des pays Anglo-Saxons ${ }^{75}$ ". La mission est confiée au mois de novembre à un diplomate sans affectation replié à Lausanne, André de Kerchove de Denterghem ${ }^{76}$. En février 1941,

70. Ibid., p. 57.

71. Ibid., p. 57.

72. Ibid., p. 58.

73. Ibid., p. 59.

74. CEGES, PDK, 85, de Kerchove à Spaak, 28 août 1942.

75. AGR, PML, 500, de Kerchove à Ryckmans, 2 octobre 1941, p. 1.

76. CEGES, PDK, 48, Spaak à de Kerchove, 27 novembre 1940. CEGES, PDK, 108, Le ravitaillement de la Belgique par l'Europe au cours de la guerre. 1941-1945. Rapport présenté par Henri Crener et le comte de Kerchove de Denterghem, 1945, p. 4. 
il préside le Comité de coordination du ravitaillement de la Belgique par l'Europe qui n'a pas d'existence juridique ou légale. Celui-ci est chargé de centraliser toutes les sommes envoyées par les Belges de l'extérieur et celles allouées par le gouvernement de Londres afin de les distribuer aux différents organismes d'exécution ${ }^{77}$. D'avril 1941 à septembre 1942, date de la fin de ses activités, l'Aide médicale aura procédé à l'achat de vivres pour une somme de 165 millions d'escudos représentant un poids brut de quelque 17000 t. Les vivres expédiés en quarante convois sont composés principalement de poissons salés ou pressés (13000 t), de conserves de poissons et de tomates $(2600 \mathrm{t})$, de figues sèches et marmelade (1 $300 \mathrm{t})$ et de produits riches en vitamines ${ }^{78}(800 \mathrm{t})^{79}$.

À partir de septembre 1942, la firme mandataire portugaise établit un programme d'achats mensuels sur la base des directives établies par le Comité de coordination de Lisbonne ${ }^{80}$. À l'automne 1943, ses exportations atteignent plus de 35000 t. Les aliments expédiés sont surtout composés de poissons ${ }^{81}$. De 1941 à 1944, les achats gouvernementaux effectués au Portugal sous le couvert du Comité de coordination de Lisbonne s'élèvent à plus d'un demi-milliard d'escudos ${ }^{82}$. L'investissement gouvernemental est comparable à celui de son homologue au Havre au cours de la Grande Guerre. Le ravitaillement du pays occupé constitue plus du tiers du total des dépenses de guerre assumées par le gouvernement en exil $^{83}$. Le Portugal assure près de $80 \%$ des envois gouvernementaux ${ }^{84}$. Au mois de décembre 1943, l'aide londonienne " en faveur des pauvres et des misérables " a bénéficié à 1300000 femmes et enfants sur un total de 8 millions de Belges occupés, majoritairement issus du monde ouvrier et de la petite bourgeoisie déclassée : " petits rentiers, petits fonctionnaires, retraités, etc. ${ }^{85}$ ". Ce maigre ravitaillement à haute valeur nutritionnelle fournit notamment, depuis le Portugal, les écœurantes cuillerées d'huile de

77. AGR, PML, 500, de Kerchove à Ryckmans, 2 octobre 1941.

78. À savoir la trinité vitaminée « huile de foie de poisson-Hepatum-citron ».

79. R. Reding, Le ravitaillement de la Belgique, op. cit., p. 69.

80. AGR, PML, 500, discours de Kerchove, décembre 1943, p. 6.

81. CEGES, PDK, 108, Le ravitaillement de la Belgique par l'Europe, p. 27 et 62.

82. Ibid., p. 57.

83. Compte rendu analytique, Sénat et Chambres réunis, 19 septembre 1944, p. 12.

84. AGR, PML, 500, discours de Kerchove, décembre 1943, p. 4. CEGES, PDK, 108, Le ravitaillement de la Belgique par l'Europe, p. 56.

85. Ibid. 
foie de morue infligées aux petits Belges sous-alimentés. Ceux-ci, plus de 70 années après la guerre, s’en souviennent encore.

Aucune des nations occupées, à l'exception notoire de la Grèce décimée par une famine, n'obtint pourtant ce que le petit mais " riche " gouvernement belge aux atours congolais arrache finalement aux Britanniques à force d'entêtement : une brèche effective et durable dans le blocus maritime pour assurer un "ravitaillement de guerre " collectif, aussi limité soit-il. Si les Britanniques se sont trompés en invoquant le détournement possible du Relief transatlantique, ils ont raison de considérer que tout ravitaillement de guerre supporterait la politique pragmatique de production prônée par un groupe informel de notables principalement issus des milieux financiers et industriels - à savoir le Comité Galopin ${ }^{86}$ - et donc l'effort de guerre ennemi. Le ravitaillement organisé à l'intérieur du blocus allié par le Gouvernement de Londres dédouane la puissance occupante d'une partie de la charge du ravitaillement destiné aux catégories les plus fragiles. Comme lors de la première occupation, l'Allemagne ne détourne pas l'aide humanitaire en provenance du Portugal mais poursuit imperturbablement ses prélèvements sur les maigres ressources indigènes.

\section{Une " politique d'abondance " en temps de pénurie mondiale (1944-1948)}

Dès la seconde Libération, les gouvernements belges successifs s'attachent à la restauration des forces de travail épuisées par quatre longues années d'occupation. Cette restauration du " capital physique et moral ${ }^{87}$ " du pays est un corollaire indispensable à la reprise de l'activité économique au même titre que la défense d'un franc fort. Ces objectifs alimentaires et monétaires ont été assignés, en exil à Londres et dans Bruxelles occupée, par les élites dirigeantes - qu'elles soient politiques, économiques ou syndicales - et ce, dans une rare unanimité. Le parti communiste qui veut participer au pouvoir y adhère dès le mois de septembre $1944^{88}$.

\footnotetext{
86. Alexandre Galopin est le gouverneur de la Société générale de Belgique. Cette holding contrôle avec ses 800 entreprises 30 à $40 \%$ du patrimoine industriel belge. Alexandre Galopin est exécuté au mois de mars 1944 par des sicaires de la SS flamande. Patrick Nefors, La collaboration industrielle en Belgique 1940-1945, Bruxelles, Éditions Racine, 2006, p. 14.

87. CEGES, PR, 179, Heymans à Richard, Lisbonne, 3 octobre 1941.

88. L. A. Bernardo y Garcia, Le Ventre des Belges, op. cit., p. 295-306.
} 
Grâce à ses prestations massives aux armées alliées depuis la Libération et le remboursement inespéré d'une partie de celles-ci, la Belgique est en mesure de reprendre dès la fin de la Seconde Guerre mondiale en Europe ses exportations de produits semi-finis et autres matériaux de construction répondant à bon prix à une demande insatiable des pays limitrophes ${ }^{89}$. Au début de l'année 1947, la production industrielle belge rattrape son niveau d'avant-guerre alors que les économies de la Grande-Bretagne, de la France ou encore des Pays-Bas demeurent à la traîne ${ }^{90}$. L'Allemagne éliminée provisoirement du jeu, la Belgique devient un éphémère pilier de l'économie de l'Europe occidentale en reconstruction. En 1947, ses réserves en devises faibles (livre sterling, florins, francs français, couronnes danoise et norvégienne) atteignent l'équivalent de 12 milliards de francs belges. Début 1948, la Belgique a consenti aux autres pays d'Europe des crédits pour un montant total de 12 à 14 milliards de francs ${ }^{91}$.

Ses abondantes réserves en devises fortes et faibles permettent à la Belgique de financer ses approvisionnements extérieurs. Cette "politique d'abondance »- de l'offre légale s'entend - constitue l'une des principales singularités de la politique alimentaire de la Belgique du second immédiat après-guerre. Le droit à l'alimentation des ouvriers en produits de base, défendu par la puissance publique, est contrebalancé par une large et précoce offre légale aux " économiquement forts ${ }^{92}$ " de produits de luxe indigènes et importés. D’après le ministre des Affaires économiques, Jean Duvieusart, l'offre de produits de luxe est destinée à détourner le " pouvoir d'achat disponible " des produits de première nécessitép ${ }^{33}$. En d'autres

89. Bernard Hanin, La politique économique des gouvernements Van Acker et Huysmans (31 mars 1946 au 12 mars 1947, Université catholique de Louvain, 1976, p. 108 (travail de fin d'études inédit). AGR, Cabinets des ministres des Affaires économiques (désormais AFEC), 82, Supreme Headquarters Allied Expeditionary Force Mission (Belgium). Supplement to Fortnightly Report. A Statement of Mutual Aid between the Allies and Belgium, mai 1945.

90. Ginette Kurgan-Van HentenryK, "Milward (Alan S.) The Reconstruction of Western Europe 1945-51 ", Revue belge de Philologie et d'Histoire, année 1986, vol. 64, n 2, p. 320 pagination de l'article ?; Jean-Charles SNOY ET D'Oppuers, La position de la Belgique dans la reconstruction économique de l'Europe et du Monde, Extrait des Annales de Sciences économiques appliquées, $\mathrm{n}^{\circ} 2$, juin 1948, p. 166.

91. Ibid., p. 171.

92. Rapport présenté par le Gouverneur, au nom du conseil de Régence sur les opérations de l'année 1946 et Rapport au Collège des Censeurs, Bruxelles, 1947, p. 44.

93. Jean-Charles SnOy et D'Oppuers, Deux années de transition vers l'économie de paix, Bruxelles, Jacobs, 1946, p. 22. 
termes, la consommation de produits de luxe était « un moyen indirect de soulager la pression inflatoire, qui autrement se serait concentrée sur les biens les plus indispensables ${ }^{94}$ ». Elle permet aux classes aisées de restaurer commodément leur train de vie malmené par l'occupation sans recourir exclusivement au marché noir. En 1947, la Commission du ravitaillement et des importations du Sénat constate que le « large esprit de compréhension " des classes laborieuses depuis la Libération n'existe pas à un même degré « chez tous ceux qui possèdent les moyens de production, de circulation et de crédit ». Celles-ci constituent les "éternelles sacrifiées ${ }^{95}$ ".

Sans doute, cette politique belge d'abondance de l'offre légale des produits de luxe ${ }^{96}$ et une répression ambivalente et inéquitable du marché noir ne s'embarrassent pas d'un discours puritain et austère comme en GrandeBretagne $^{97}$. Ce ravitaillement à deux vitesses complique voire rend impossible l'adhésion sinon le consentement de la demande ouvrière et de l'offre alimentaire aux sacrifices demandés au nom de la restauration du pays. Dans le processus de reconstruction de l'Europe occidentale du second immédiat après-guerre, la politique alimentaire personnifie la singularité du cas belge mais aussi ses contradictions. Elle se démarque des choix opérés par ses voisins français, néerlandais ou britannique impécunieux mais obsédés par la modernisation de leurs appareils de production respectifs. En temps de pénurie mondiale, une Belgique solvable opte à la fois pour un "franc fort ", une politique d'abondance de l'offre y compris de produits de luxe, un processus hâtif de libération de la production agricole régulée et un rejet de toute planification des investissements ou de la production industrielle.

Ce " miracle belge ${ }^{98}$ " célébré par la presse nationale et internationale ne s'accomplira pas dans les assiettes de l'ouvrier belge. À l'image du premier immédiat après-guerre, la restauration économique du pays, entre septembre 1944 et décembre 1948, repose sur une répartition inéquitable des sacrifices demandés par les élites dirigeantes et des bénéfices engrangés.

94. AGR, AFEC, 1983, Jean Duvieusart, La situation économique de la Belgique, s.d. [novembre 1948], p. 3.

95. Documents parlementaires, Sénat, Budget, Ravitaillement, Exercice 1947, 4 juin 1947, p. 3. 96. AGR, CABRAV, 1583, Note à Monsieur le secrétaire général, Bruxelles, 9 juillet 1947, p. 2. 97. AGR, CABRAV, 1630, Revue de presse, n 128, 16 février 1945, p. 7 ; Revue de presse, $n^{\circ} 119$, 6 février 1945 , p. 6.

98. J.-C. Snoy et D’Oppuers, Deux années de transition, op. cit., p. 169. 
La libération progressive du commerce de certains produits alimentaires de base bénéficie surtout aux classes aisées qui se détournent progressivement du marché noir après l'augmentation de l'offre sur le marché légal. Quant aux " économiquement faibles" ", des rations supplémentaires pour les travailleurs de force, des ajustements salariaux voire des allocations ponctuelles permettent de répondre difficilement à la cherté de la vie en dépit des prix maxima et des coûteuses subventions maintenues jusqu'en 1948 pour les principales denrées alimentaires encore rationnées comme le pain, le sucre, les matières grasses et la viande. En janvier 1949, pour paraphraser l'historien américain Steven L. Kaplan étudiant le cas français, le monde ouvrier belge retrouve enfin un "pain de bonne qualité, en quantité suffisante et à un prix raisonnable ${ }^{100}$ ". La fin du rationnement marque un retour au maigre standard de vie d'avant-guerre. Il résulte surtout de la fin des allocations internationales sur les dernières denrées encore rationnées. Elle ne signifie aucune amélioration substantielle dans l'ordinaire des ouvriers et de leur famille, confrontés en outre à la hausse persistante du coût de la vie. La société de consommation de masse est encore à venir.

\section{Conclusion}

Aux yeux des partis mais aussi des syndicats ouvriers traditionnels belges, les élites financières et industrielles du pays détiennent les clés de la reconstruction économique et donc de sa normalisation politique. S'appuyant sur une puissance publique faible mais prodigue, le monde des affaires sait donner la preuve d'une remarquable capacité d'adaptation et de réaction face à la menace révolutionnaire puis communiste au cours des deux libérations ou celle d'une radicalisation syndicale des forces de travail aprèsguerre. Les principaux objectifs assignés en temps de crise alimentaire par les élites dirigeantes belges - dont fait partie intégrante le cadre socialiste depuis 1918 - sont effectivement remplis. Au cours des deux occupations, la population échappe à la famine en dépit du blocus britannique et des prédations allemandes massives nourries par un marché noir florissant ${ }^{101}$.

99. Archives de l'État à Bruges, papiers Achiel Van Acker, 428, discours radiophonique de Van Acker, s.d. [décembre 1945], p. 2.

100. Steven L. Kaplan, Le pain maudit. Retour sur la France des années oubliées 1945-1958, Paris, Fayard, 2008, p. 47.

101. Au cours de la Seconde Guerre mondiale, l'historien néerlandais Hein A. M. Klemann estime jusqu’à $30 \%$ la moyenne des détournements dans la plupart des pays occupés d'Europe occidentale, 
Le régime démocratique capitaliste et l'Establishment sortent renforcés de l'épreuve de guerre et de sortie de guerre. Au cours des deux après-guerres, les forces de travail restaurées participent sans trop de remous à la reconstruction économique du pays au prix d'une coûteuse politique alimentaire aux côtés de réformes politiques, sociales et fiscales. Ces réformes ne remettent pas en question l'ordre établi. Elles assurent, voire renforcent, la position du cadre socialiste réformiste au sein du monde ouvrier.

\section{L'auteur}

Docteur en histoire de l'Université libre de Bruxelles et chef de travaux aux Archives générales du Royaume, Luis Angel Bernardo y Garcia coordonne le transfert des archives coloniales. Il a focalisé ses inventaires et recherches sur la Seconde Guerre mondiale, y compris l'exil du gouvernement belge à Londres, et sur le second immédiat après-guerre, notamment les aspects économique, alimentaire et militaire. II a publié Le Ventre des Belges. Une histoire alimentaire des temps d'occupation et de sortie de guerre (1914-1921 et 1939-1948), Bruxelles, Archives générales du Royaume, 2017. Contact : luis_angel.bernardo_y_garcia@ arch.be

donnée statistique qui ne transparait pas dans les statistiques officielles. En Belgique, le marché noir détourne $35 \%$, voire $50 \%$, de la production agricole indigène. Hein A. M. KLEMANN, «Waarom honger in Europa in de Tweede Wereldoorlog ? ", in Madelon de Keizer, Mariska Heijmans-Van Bruggen, Erik Somers et Angela Van Son (dir.) Thuisfront. Oorlog en economie in de twintigste eeuw. Veertiende jaarboek van het Nederlands Instituut voor Oorlogsdocumentatie, Zutphen, Walburg Pers, 2003, p. 113-125. 